EPJ Web of Conferences 60, 20033 (2013)

DOI: $10.1051 /$ epjconf/20136020033

(C) Owned by the authors, published by EDP Sciences, 2013

\title{
Searches for Heavy Lepton Partners of Neutrinos in Proton-Proton Collisions at CMS Experiment in the Context of Seesaw Mechanism
}

\author{
S.Vanini ${ }^{3}$, A. Gozzelino ${ }^{1,3}$, P.Checchia ${ }^{2}$, E.Torassa ${ }^{2}$ \\ ${ }^{1}$ INFN, Laboratori Nazionali di Legnaro, Legnaro (Padova), Italy. \\ ${ }^{2}$ INFN, Sezione di Padova, Padova, Italy. \\ ${ }^{3}$ Dipartimento di Fisica e Astronomia dell'Università di Padova, Padova, Italy.
}

\begin{abstract}
A search is presented in proton-proton collisions at $\sqrt{s}_{\mathrm{s}}=7 \mathrm{TeV}$ for fermionic triplet states expected in type III seesaw models. The search is performed using final states with three isolated charged leptons and an imbalance in transverse momentum. The data, collected with the CMS detector at the LHC, correspond to an integrated luminosity of $4.9 \mathrm{fb}^{-1}$. No excess of events is observed above the background predicted by the standard model, and the results are interpreted in terms of limits on production cross sections and masses of the heavy partners of the neutrinos in type III seesaw models. Depending on the considered scenarios, lower limits are obtained on the mass of the heavy partner of the neutrino that range from 180 to $210 \mathrm{GeV}$. These are the first limits on the production of type III seesaw fermionic triplet states reported by an experiment at the LHC.
\end{abstract}

\section{Introduction}

Ideas beyond the standard model have been proposed to accommodate the finite values of neutrino masses inferred from neutrino oscillations. An interesting possibility is provided by the seesaw mechanism, in which a small Majorana mass can be generated for each of the known neutrinos by introducing massive states with Yukawa couplings to leptons and to the Higgs field. Seesaw models called type I, type II, and type III introduce heavy states of mass $\mathrm{M}$, that involve, respectively, weak-isospin singlets, scalar triplets, and fermion triplets (Ref. [1]).

At the Large Hadron Collider (LHC), type II and III states can be produced through gauge interactions, so that the possible smallness of the Yukawa couplings does not affect the production cross section of the heavy states. In particular, the possibility of discovering a type III fermion at LHC is promising with early data.

This paper reports on a search for fermionic triplet states $(\Sigma)$ expected in type III seesaw models, in final states with three charged leptons and an imbalance in transverse momentum. The data sample corresponds to an integrated luminosity of $4.9 \mathrm{fb}^{-1}$, collected in proton-proton collisions at $\sqrt{\mathrm{S}}_{\mathrm{S}}=7 \mathrm{TeV}$ with the Compact Muon Solenoid (CMS) detector at the LHC in 2011. The analysis is based on the model described in Ref. [2], using the implementation of Ref. [3]. Three possibilities are considered for the mixing matrix element for the leptons: equal mixings i.e. the flavor-democratic scenario

\footnotetext{
a Corresponding author: sara.vanini@pd.infn.it
}

(FDS); the muon scenario $(\mu \mathrm{S})$ and the electron scenario (eS).

\section{CMS Experiment and Physics Objects}

A detailed description of the CMS detector can be found in Ref. [4]. The central feature of the CMS apparatus is a super conducting solenoid that provides an axial magnetic field of $3.8 \mathrm{~T}$. A silicon tracker, a lead-tungstate crystal electromagnetic calorimeter (ECAL), and a brass scintillator hadron calorimeter (HCAL) reside within the magnetic field volume. Muons are identified using the central tracker and a muon system consisting of gas ionization detectors embedded in the steel return yoke outside of the solenoid.

All objects are reconstructed using a particle-flow (PF) algorithm [5]. The PF algorithm combines information from all subdetectors to identify and reconstruct particles detected in the collision, namely charged hadrons, photons, neutral hadrons, muons, and electrons. Jets are reconstructed using the anti-kT jet clustering algorithm with a distance parameter of 0.5 , and jet energies are corrected for non-uniformity in calorimeter response and for differences found between jets in simulation and in data. An imbalance in transverse momentum $\left(\mathrm{E}_{\mathrm{T}}^{\text {miss }}\right)$ is defined by the magnitude of the vectorial sum of the transverse momenta $\left(\mathrm{p}_{\mathrm{T}}\right)$ of all particles reconstructed through the PF algorithm.

Seesaw events are generated using the FeynRules and MadGraph computer programs, parton showers and hadronization are implemented using the pythia generator 
(v6.420). The detector simulation is based on the Geant4 program.

\section{Event Selection}

Events are selected through two-lepton triggers: two muons, two electrons, or one electron and one muon are required. The selected events must contain at least two lepton candidates with trajectories that have a transverse impact parameter of less than $0.2 \mathrm{~mm}$ relative to the principal interaction vertex.

Muon candidates are reconstructed from a fit performed to hits in both the silicon tracker and the outer muon detectors. The specific selection requirements for a muon are: (i) $\mathrm{p}_{\mathrm{T}}>10 \mathrm{GeV}$, (ii) $|\eta|<2.4$, (iii) more than 10 hits in the silicon tracker, and (iv) a global-muon fit with $\chi / \mathrm{dof}<10$, where dof is the number of degrees of freedom. Electron candidates are reconstructed using clusters of energy depositions in the ECAL that match the extrapolation of a reconstructed track. The specific requirements for a reconstructed electron are: (i) $\mathrm{p}_{\mathrm{T}}>10$ $\mathrm{GeV}$, (ii) $|\eta|<1.44$, within the fully instrumented part of the central barrel, or $1.57<|\eta|<2.5$ for the endcap regions, (iii) not being a candidate for photon conversion, and (iv) the tracks reconstructed using three independent algorithms to give the same sign for the electric charge.

\section{Backgrounds}

Three types of SM processes can produce a three-lepton final state: (i) events containing three or more prompt leptons from production and leptonic decays of two or three electroweak (EW) bosons (irreducible background); (ii) $\mathrm{V}+\gamma$ and $\mathrm{V}+\gamma^{*}$ events, where $\mathrm{V}$ represents any $\mathrm{EW}$ boson, with the accompanying photons converting to $1^{+} 1^{-}$; and (iii) events with one or two prompt leptons and additional non-prompt leptons that arise from leptonic decays of hadrons within jets, called "misidentified jets". (i) The irreducible background is dominated by $\mathrm{SM} \mathrm{W}^{+} Z$ production, but also includes $\mathrm{ZZ}$ and three-boson events. They are evaluated using MC simulation.

(ii) Photon conversions in the presence of $\mathrm{W}$ or $\mathrm{Z}$ bosons can produce isolated leptons that constitute another source of background. External conversions of photons (produced photons that interact with the material in the detector to yield primarily $\mathrm{e}^{+} \mathrm{e}^{-}$pairs), are evaluated from simulation. Internal conversions, involving the direct materialisation of virtual photons into $\mu^{+} \mu^{-}$or $\mathrm{e}^{+} \mathrm{e}^{-}$pairs, can also provide a similar source of background. Both external and internal conversions can become problematic when one of the two final state leptons carries off most of the photon energy, and the second lepton is not detected. The contribution from internal photon conversions is evaluated with data-driven techniques.

(iii) The largest background, aside from the irreducible backgrounds, arises from the $\mathrm{Z}+\mathrm{jets}$ process (including the Drell-Yan contribution), in which the $\mathrm{Z}$ boson decays leptonically, and a jet in the event is misidentified as a third lepton. Processes with non-prompt leptons from heavy-flavour decays are not simulated with sufficient accuracy with the MC generators and we therefore use a method based on data to estimate this contribution.
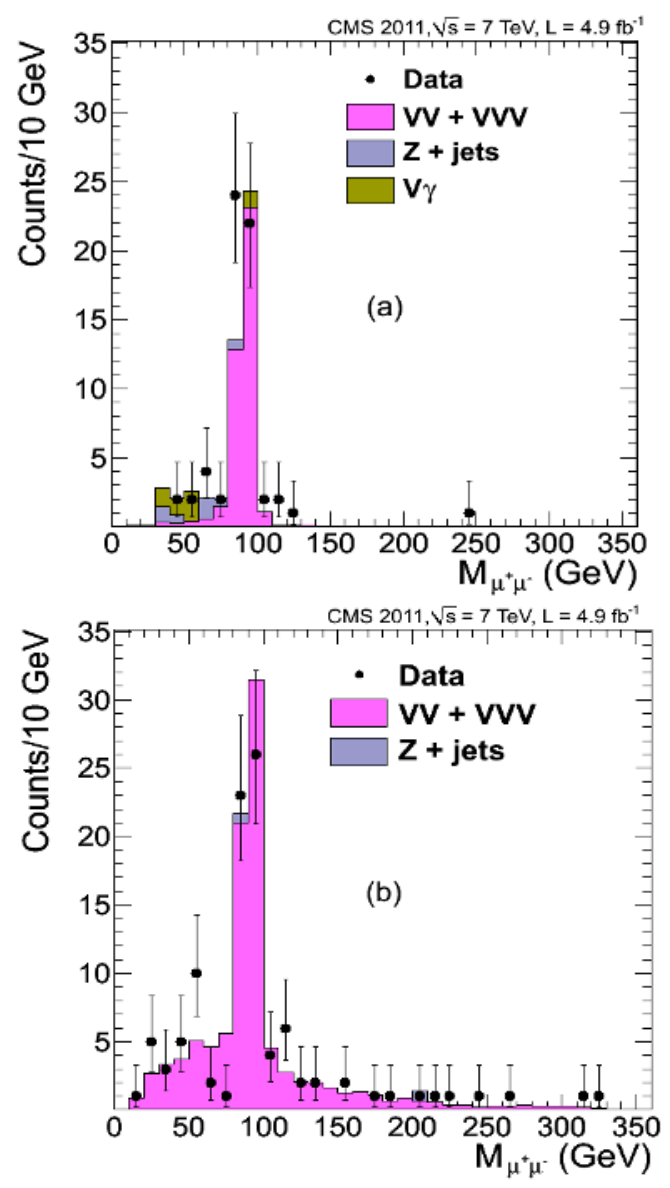

Figure 1. Distributions of the $\mu-\mu+$ invariant mass for (a) $\mu^{-} \mathrm{e}^{+} \mu^{+}$and (b) $\mu^{-} \mu^{+} \mu^{+}$events in data (black points) and all major SM backgrounds, before applying any requirement on the $\mu^{-} \mu^{+}$mass to reject $Z$ bosons.

\section{Analysis}

The candidate events used for the search are required to have: (i) three isolated charged leptons originating from the same primary vertex, (ii) sum of the lepton charges equal to +1 , (iii) $\mathrm{E}_{\mathrm{T}}{ }^{\text {miss }}>30 \mathrm{GeV}$, (iv) $\mathrm{p}_{\mathrm{T}}>18,15,10 \mathrm{GeV}$ for the lepton of highest, next-to-highest, and lowest $\mathrm{p}_{\mathrm{T}}$, and (v) $\mathrm{H}_{\mathrm{T}}<100 \mathrm{GeV}$, where $\mathrm{H}_{\mathrm{T}}$ is the scalar sum of the transverse momenta of jets with $\mathrm{p}_{\mathrm{T}}>30 \mathrm{GeV}$ and $|\eta|<2.4$. The selected events are classified into six categories that depend on lepton flavour and electric charge: $\mu^{-} \mathrm{e}^{+} \mathrm{e}^{+}$, $\mu^{-} \mathrm{e}^{+} \mu^{+}, \mu^{-} \mu^{+} \mu^{+}, \mathrm{e}^{-} \mu^{+} \mu^{+}, \mathrm{e}^{-} \mathrm{e}^{+} \mu^{+}$, and $\mathrm{e}^{-} \mathrm{e}^{+} \mathrm{e}^{+}$. Fig. 1 shows the distributions of the $\mu^{-} \mu^{+}$invariant mass for $\mu^{-} \mathrm{e}^{+} \mu^{+}$and $\mu^{-} \mu^{+} \mu^{+}$events in data, before applying any requirement on the $\mu^{-} \mu^{+}$mass, compared to the sum of SM background contributions. A peak in the $\mu^{-} \mu^{+}$effective mass close to that of the $\mathrm{Z}$ boson is evident in both simulated events and in data.

To reduce the background from $\mathrm{W}^{+} \mathrm{Z}$ events, a $\mathrm{Z}$ veto is added to the selection requirements for the corresponding categories: events with at least one mass combination in the range $[82,102] \mathrm{GeV}$ are rejected. 

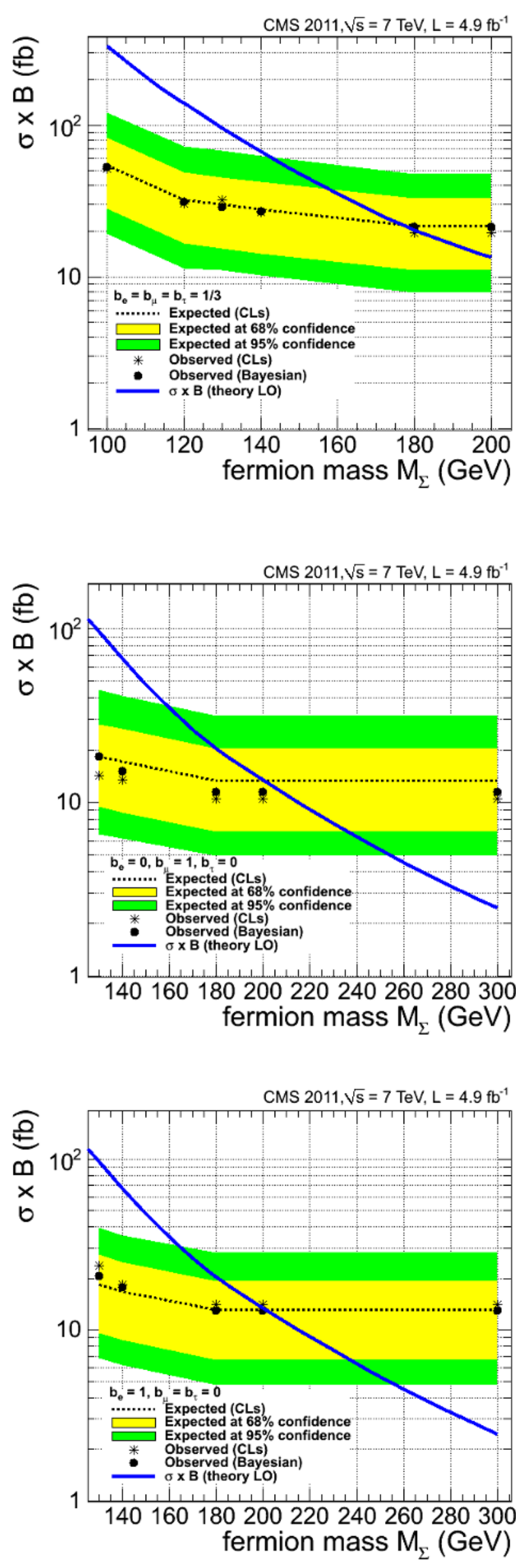

Figure 2. The expected (dashed line) and observed (asterisks and black points) exclusion limits at $95 \%$ confidence level on $\sigma \mathrm{xB}$ as a function of the fermion mass $\mathrm{M}_{\Sigma}$, for the tree mixing scenarios. The solid (blue) curve represents the predictions of the LO type III seesaw models. The light (yellow) and dark (green) shaded areas represent, respectively, the 1 standard deviation and 2 standard deviations limits on the expected results obtained from MC pseudo-experiments. The asterisks and the black points show, respectively, the observed limits computed following a frequentist method based on the CLs criterion and a Bayesian approach.

To reject lepton pairs from decays of heavy-flavour quarks, events with $m_{\Perp}<12 \mathrm{GeV}$ are also discarded.

Other sources of background in final states with three leptons arise from conversions photons into additional lepton pairs, therefore an additional $\mathrm{Z}$ veto is applied to the $\mu^{-} \mathrm{e}^{+} \mu^{+}$and $\mathrm{e}^{-} \mathrm{e}^{+} \mathrm{e}^{+}$categories: events with three leptons invariant mass in the range $[82,102] \mathrm{GeV}$ are rejected.

\section{Results and Conclusions}

No significant excess of events is observed relative to the SM expectations in any of the six channels. Combining all channels, we set upper limits at the $95 \%$ confidence level $(\mathrm{CL})$ on $\sigma \times \mathrm{B}$, the product of the production cross section of $\Sigma^{+} \Sigma^{0}$ and its branching fraction (B) to the three-lepton final states. $\mathrm{B}$ depends on $\mathrm{M}_{\Sigma}$. The upper limits on $\sigma \times B$ as a function of fermion mass $M_{\Sigma}$, combining for all channels by multiplying the corresponding like-lihood functions, are shown in Fig. 2 for FDS, $\mu \mathrm{S}$, and eS possibilities. The dashed lines correspond to the expected limits obtained from MC pseudo-experiments. The observed limits on data are computed following both a Bayesian approach and a frequentist method also based on the CLs criterion. In both calculations, the uncertainties on efficiencies for detecting signal, the uncertainty on integrated luminosity and on the expected SM background, are treated as uninteresting "nuisance" parameters with Gaussian or log-normal densities. Finally, we extract lower limits on $\mathrm{M}_{\Sigma}$ using the theoretical dependence of the cross section on $\mathrm{M}_{\Sigma}$, as represented by the solid blue lines of Fig. 2, for the three possibilities for the type III seesaw model for signal (Ref. [6]).

\section{References}

1. T.P. Cheng, L.-F. Li, Phys. Rev. D 22 (1980) 2860, http://dx.doi.org/10.1103/PhysRevD.22.2860

2. F. del Aguila, J.A. Aguilar-Saavedra, Nucl. Phys. B 813 (2009) 22, arXiv:0808.2468, http://dx.doi.org/10.1016/j.nuclphysb.2008.12.029

3. C. Biggio, F. Bonnet, Eur. Phys. J. C 72 (2012) 1899, arXiv:1107.3463, http://dx.doi.org/10.1140/epjc/s10052-012-1899-z

4. S. Chatrchyan, et al., JINST 03 (2008) S08004, http://dx.doi.org/10.1088/1748-0221/3/08/S08004

5. CMS Collaboration, CMS-PAS-PFT-09-001, URL http://cdsweb.cern.ch/record/1194487, 2009.

6. CMS Collaboration, Phys. Lett. B 718 (2012) 348-368. 